\title{
Descriptive analysis of long COVID sequelae identified in a multidisciplinary clinic serving hospitalised and non- hospitalised patients
}

\author{
Stine Johnsen $\mathbb{1}^{1,12}$, Stefan M. Sattler ${ }^{1,2,12}$, Kamilla Woznica Miskowiak ${ }^{3}$, Keerthana Kunalan ${ }^{1}$, Alan Victor ${ }^{1}$, \\ Lars Pedersen $\mathbb{D}^{1}$, Helle Frost Andreassen ${ }^{1}$, Barbara Jolanta Jørgensen ${ }^{4}$, Hanne Heebøll ${ }^{4}$, \\ Michael Brun Andersen ${ }^{5}$, Lisbeth Marner ${ }^{6}$, Carsten Hædersdal ${ }^{6}$, Henrik Hansen ${ }^{7,8}$, Sisse Bolm Ditlev ${ }^{7,9}$, \\ Celeste Porsbjerg ${ }^{1,7}$ and Thérèse S. Lapperre (10 ${ }^{1,10,11}$
}

\begin{abstract}
${ }^{1}$ Dept of Respiratory Medicine, Copenhagen University Hospital at Bispebjerg, Copenhagen, Denmark. ${ }^{2}$ Biomedical Institute, Copenhagen University, Copenhagen, Denmark. ${ }^{3}$ Neurocognition and Emotion in Affective Disorders, Copenhagen, Denmark. ${ }^{4}$ Dept of Radiology, Copenhagen University Hospital at Bispebjerg, Copenhagen, Denmark. ${ }^{5}$ Dept of Radiology, Copenhagen University Hospital at Herlev/Gentofte, Copenhagen, Denmark. ${ }^{6}$ Dept of Clinical Physiology and Nuclear Medicine, Copenhagen University Hospital at Bispebjerg and Frederiksberg, Copenhagen, Denmark. ${ }^{7}$ Respiratory Research Unit, Dept of Respiratory Medicine, Copenhagen University Hospital at Bispebjerg, Copenhagen, Denmark. ${ }^{8}$ Respiratory Research Unit, Dept of Respiratory Medicine, Copenhagen University Hospital at Hvidovre, Copenhagen, Denmark. ${ }^{9}$ Copenhagen Center for Translational Research, Copenhagen University Hospital at Bispebjerg, Copenhagen, Denmark. ${ }^{10}$ Dept of Pulmonology, University Hospital Antwerp, Antwerp, Belgium. ${ }^{11}$ Laboratory of Experimental Medicine and Paediatrics, University of Antwerp, Antwerp, Belgium. ${ }^{12}$ These authors contributed equally.
\end{abstract}

Corresponding author: Stine Johnsen (stine.johnsen.01@regionh.dk)

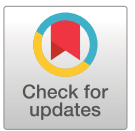

This version is distributed under the terms of the Creative Commons Attribution NonCommercial Licence 4.0. For commercial reproduction rights and permissions contact permissions@ersnet.org

This article has supplementary material available from openres.ersjournals.com

Received: 20 March 2021 Accepted: 20 April 2021

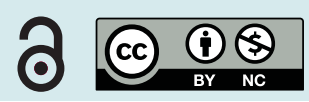

Shareable abstract (@ERSpublications)

3 months post-COVID-19, most patients have at least one organ function impairment. All patients have some degree of cognitive impairment and abnormal dyspnoea symptom scores. Abnormal HRCT and impaired $D_{\text {LCO }}$ are predominately found in inpatients. \#longCOVID https://bit.ly/2QB2j0J

Cite this article as: Johnsen S, Sattler SM, Miskowiak KW, et al. Descriptive analysis of long COVID sequelae identified in a multidisciplinary clinic serving hospitalised and non-hospitalised patients. ERJ Open Res 2021; 7: 00205-2021 [DOI: 10.1183/23120541.00205-2021].

\section{Abstract}

Background There are emerging data of long-term effects of coronavirus disease 2019 (COVID-19) comprising a diversity of symptoms. The aim of this study was to systematically describe and measure pulmonary and extra-pulmonary post-COVID-19 complications in relation to acute COVID-19 severity.

Methods Patients attending a standard of care 3 months post-hospitalisation follow-up visit and those referred by their general practitioner because of persistent post-COVID-19 symptoms were included. Patients underwent symptomatic, quality of life, pulmonary (lung function and high-resolution computed tomography (HRCT)), cardiac (high-resolution ECG), physical (1-min sit and stand test (1-MSTST), handgrip strength, cardiopulmonary exercise testing (CPET)) and cognitive evaluations.

Results All 34 hospitalised and 22 out of 23 non-hospitalised patients had $\geqslant 1$ complaint or abnormal finding at follow-up. Overall, 67\% of patients were symptomatic (Medical Research Council (MRC) $\geqslant 2$ or COPD assessment test (CAT) $\geqslant 10$ ), with no difference between hospitalised versus non-hospitalised patients. Pulmonary function (forced expiratory volume in $1 \mathrm{~s}\left(\mathrm{FEV}_{1}\right)$ or diffusing capacity of the lung for carbon monoxide $\left.\left(D_{\text {LCO }}\right)\right)<80 \%$ of predicted) was impaired in $68 \%$ of patients. $D_{\text {LCO }}$ was significantly lower in those hospitalised compared to non-hospitalised $(70.1 \pm 18.0$ versus $80.2 \pm 11.2 \%$ predicted, $\mathrm{p}=0.02$ ). Overall, 53\% had an abnormal HRCT (predominantly ground-glass opacities) with higher composite computed tomography (CT) scores in hospitalised versus non-hospitalised patients (2.3 (0.14.8 ) and $0.0(0.0-0.3), \mathrm{p}<0.001)$. 1-MSTST was below the 25th percentile in almost half of patients, but no signs of cardiac dysfunction were found. Cognitive impairments were present in 59-66\% of hospitalised and $31-44 \%$ of non-hospitalised patients $(\mathrm{p}=0.08)$.

Conclusion Three months after COVID-19 infection, patients were still symptomatic and demonstrated objective respiratory, functional, radiological and cognitive abnormalities, which were more prominent in hospitalised patients. Our study underlines the importance of multidimensional management strategies in these patients. 
Introduction

Without doubt the coronavirus disease 2019 (COVID-19) pandemic will cast a long shadow into the future. With increasing numbers of patients who have survived the disease, it is necessary to attain a better understanding of the long-term health complications of COVID-19. The lungs are often the first target organ of severe acute respiratory syndrome coronavirus 2 (SARS-CoV-2) infection, but we now know that the virus can spread to many different organs [1-4]. Experience from previous coronavirus outbreaks such as severe acute respiratory syndrome (SARS) and Middle East respiratory syndrome (MERS) suggest that some patients will experience long-term complications [5].

Reports of long-term effects of COVID-19, also referred to as "long COVID”, in more severe hospitalised and less severe non-hospitalised patients have begun to be published [6-15]. The predominant persistent symptoms include breathlessness, cough and fatigue. In addition, lung function and chest computed tomography (CT) changes may be impaired months after the infection [9]. There is also compiling evidence for neurological complications of COVID-19, especially among the severely affected patients, including loss of smell and taste, tingling sensations, dizziness and severe fatigue, as well as more severe outcome [16-18]. A large proportion of COVID-19 patients may also experience persistent cognitive impairments for months after their recovery [8].

The diversity of symptoms suggests both pulmonary as well as extrapulmonary involvement in long COVID. It is clearly crucial to understand not only the relative incidence of symptoms and abnormal findings from different organ systems, but also to what extent they overlap and relate to the severity of acute COVID-19. In addition, the distribution and mechanisms of post-COVID complications may differ among long COVID patients that were hospitalised and those not hospitalised during acute COVID-19. Being affected by more than one type of organ dysfunction significantly contributes to the overall burden of disease and may impact the speed at which patients return to normal life after their COVID-19 illness.

Hence, the aim of this study was to systematically examine both long-term pulmonary and extrapulmonary function in a cohort of hospitalised and non-hospitalised patients who had COVID-19 in the spring of 2020. Multidisciplinary assessment included eight different impairment domains comprising respiratory symptoms, quality of life, working ability, pulmonary function, radiological pulmonary changes, cognitive assessment, functional status and cardiac-autonomic evaluation.

\section{Methods}

This was a cross-sectional study. Diagnosis of COVID-19 was made by a positive PCR test for SARS-CoV-2 from the upper respiratory tract or a positive COVID-19 IgG titre. Patients were evaluated in the respiratory outpatient clinic 3 months after discharge (hospitalised group) or resolution of the acute disease for patients referred by their general physician on three visits, conducted over a period of maximum 4 weeks. A subgroup of patients was referred for visit including a cardiopulmonary exercise test (CPET). This study was approved by the regional ethics committee (H-20035553), and all patients gave their informed consent. Methods are reported in brief and detailed information can be found in the online supplementary material.

\section{Patient-reported outcomes}

Type and degree of respiratory symptoms were assessed using the COPD Assessment Test (CAT) and Medical Research Council (MRC) dyspnoea score (MRC). References are found in supplementary methods.

Work Productivity and Activity Impairment Questionnaire (WPAI) and the 5 Dimension 5 Level Quality of Life Questionnaire (EQ-5D-5L) [19] were obtained. In addition, the Post-COVID-19 Functional Status (PCFS) Scale [20] was completed. Subjective cognitive functions were assessed with the Cognitive Failures Questionnaire (CFQ) [21].

\section{Lung function testing}

Spirometry and diffusion capacity measurements were performed in accordance with European Respiratory Society (ERS) recommendations [22].

\section{Functional capacity and cardiopulmonary exercise testing}

Participants performed the 1-min sit-to-stand test (1-MSTST) with saturation, heart rate, Borg dyspnoea measurement and handgrip dynamometry [23]. Cardiopulmonary exercise testing (CPET) was performed in patients that were symptomatic, had an abnormal lung function and/or if high-resolution computed tomography (HRCT) scans demonstrated significant pathology. In addition, patients had to be considered 
able to perform the test and understand the instructions given. Decreased ventilatory capacity was defined as reduced peak oxygen consumption $<84 \%$ of expected according to age, sex and body height [24].

\section{High-resolution computed tomography of the chest}

HRCT images were obtained in inspiration and expiration in supine position. HRCT was analysed using a scoring system as previously suggested by SøysETH et al. [25]. Scans were reviewed in random order and in consensus by two chest radiologists. Both observers (HH and MBA) were blinded regarding clinical outcome. All items were scored from 0 to 12 (representing $0-100 \%$ of lung parenchyma) at four anatomical levels. A total score was calculated by adding the scores for each finding ranging from 0 to 96 .

\section{Assessments of cognitive function}

Objective performance-based cognitive functions were assessed with a brief $(\approx 20 \mathrm{~min})$ cognition test battery consisting of the Screen for Cognitive Impairment in Psychiatry Danish Version (SCIP-D) [26] and the Trail Making Test-Part B (TMT-B) [27]. Together, these tests measure verbal learning and memory, working memory, verbal fluency, processing speed and executive function.

\section{Statistical analysis}

Data are presented as mean \pm standard deviation if normally distributed, median (interquartile range) (non-normal distributed), or count and percentage. Intergroup comparison was performed using a one-way test if normally distributed, a Kruskal-Wallis test if non-normally distributed or a Chi square test for categorical variables. Correlation analysis was performed using Pearson's correlation coefficients. If missing values were present, they were deleted in pairs. Group comparisons of cognition data were carried out with independent t-tests or Mann-Whitney tests for normally and non-normally distributed data, respectively. In case of significant differences in demographic variables between hospitalised and non-hospitalised patients in age and sex, post hoc analyses were covaried for these variables. p-values $<0.05$ were considered statistically significant. Analyses was performed with R (Version 4.0.3).

\section{Results}

For patient flow please see figure 1. The protocol was first offered only to patients who had been admitted to the hospital with severe COVID-19 illness, but it soon became evident through social media and requests from general practitioners that a similar offer to outpatients was necessary. Unofficially it therefore became possible for general practitioners to refer patients directly to our research protocol. An overview of impairment in the different domains are depicted in figure 2.

\section{Demographics}

In table 1 we present patient demographics. Hospitalised patients were predominately male; $62 \%$ males among the hospitalised and $30 \%$ in the non-hospitalised group, respectively $(p=0.04)$, and significantly older than non-hospitalised (57 versus 41 years; $\mathrm{p}<0.001$ ). The main comorbidity was a history of asthma



FIGURE 1 Flowchart for participants recruited between March 15 and August 31 2020. *: number of Covid-positive individuals in the community unknown. ${ }^{* *}$ : all patients were offered standard of care, which included LFU, DCO, high-resolution computed tomography (HRCT), clinical evaluation, multidisciplinary team and follow-up visit with follow-up plan including any additional visit. ${ }^{* \star *}$ : only patients with dyspnoea or significant symptoms were referred from their general practitioner (GP). 
a)

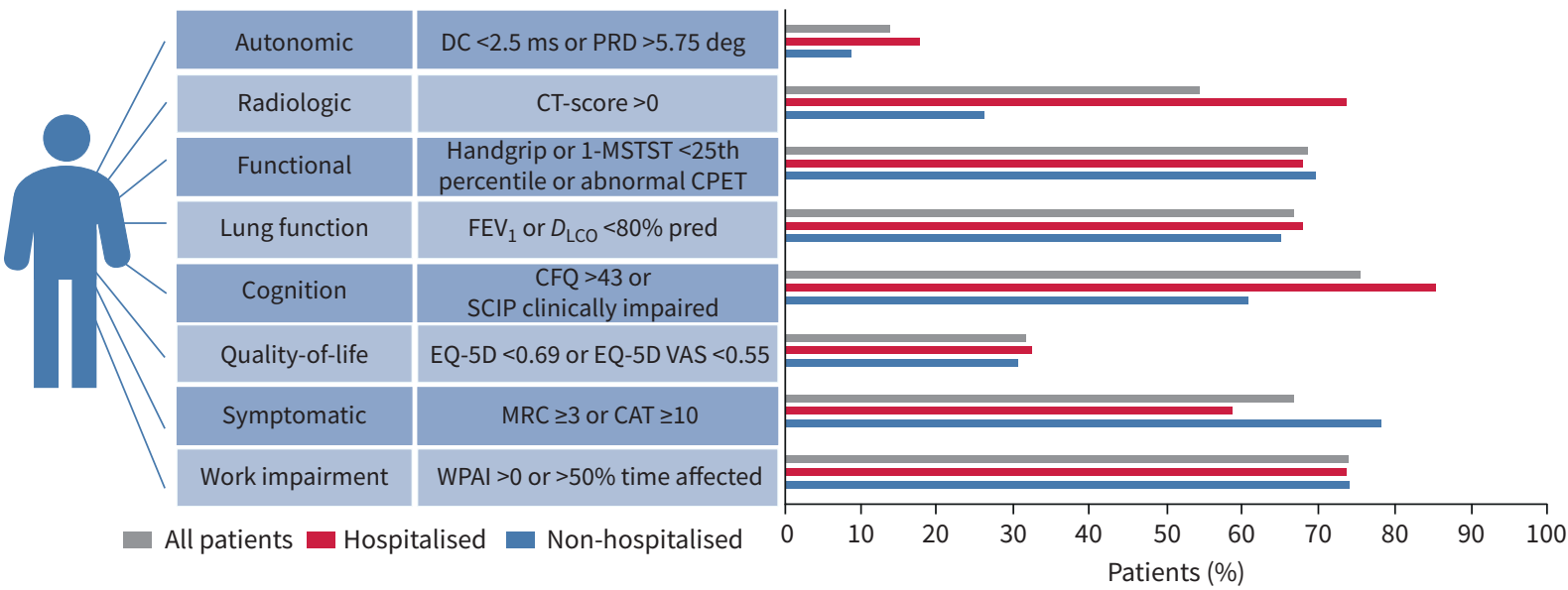

b)

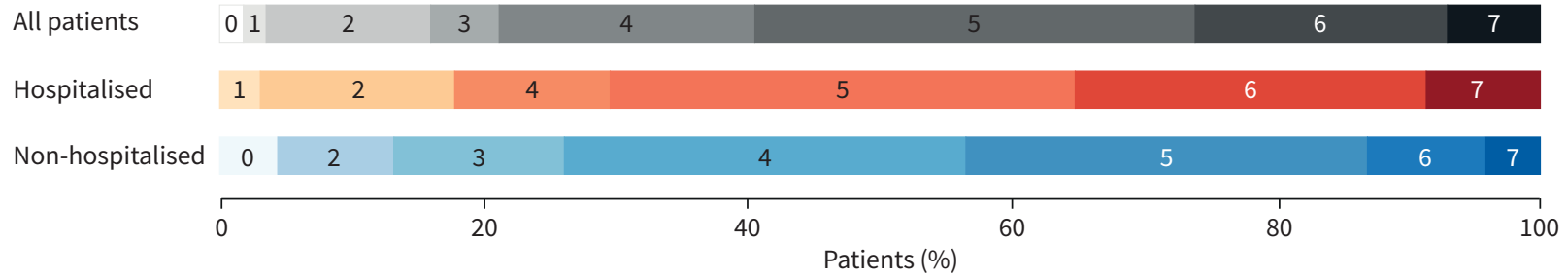

FIGURE 2 Long-term impact of coronavirus disease 2019 (COVID-19). a) Number of patients that presented with an impairment in the individual domains shown for all patients, hospitalised patients and non-hospitalised patients. b) Percentage distribution of number of impaired domains. DC: deceleration capacity; PRD: periodic repolarisation dynamics; 1-MSTST; 1-min sit and stand test; CPET: cardiopulmonary exercise testing; $\mathrm{FEV}_{1}$ : forced expiratory volume in $1 \mathrm{~s}$; $D_{\mathrm{LCO}}$ : diffusing capacity of the lung for carbon monoxide; PCFS: post-COVID functional Scale; SCIP: Screen for cognitive impairment in psychiatry; CAT: COPD Assessment Test; MRC: Medical Research Council Dyspnoea Score; CFQ: Cognitive Failures Questionnaire; CT: computed tomography; WPAl: Work Productivity and Activity Impairment Questionnaire; EQ-5D: 5 Dimension 5 Level Quality of Life Questionnaire; EQ-5D VAS: 5 Dimension 5 Level Quality of Life Questionnaire visual analog score.

in $26 \%$ of the patients. Mean body mass index (BMI) was 27.4, and 12 (26\%) patients had a BMI of $>30$. Out of the 57 patients only one was currently smoking and previous smokers and nonsmokers were evenly distributed between hospitalised and non-hospitalised groups. In table 1 data on initial COVID-19 for the 34 hospitalised patients are presented. Twenty-one of the 34 patients required oxygen therapy, and about half of all patients had oxygen delivered by high-flow. Mean duration of hospitalisation was 13 days.

\section{Patient reported outcomes}

In table 2 patient-reported outcomes at 3 months are shown. Non-hospitalised patients trended towards higher CAT scores. Overall, 67\% were symptomatic based on CAT or MRC. The median patient reported EQ5D-5 L “PRO” health status was 70 (55-81). Although not significant, the non-hospitalised patients perceived their health to be lower than the patients who had been hospitalised. Subjective self-reported cognitive difficulties on the CFQ were generally high, with $89 \%$ reporting large difficulties (scores $\geqslant 43$, corresponding to $\geqslant 1$ sD above mean of healthy controls [21]).

\section{Lung function tests and functional status tests}

In table 3 we present lung function data and functional status data assessed at the 3-month follow-up. Overall, $68 \%$ of patients had reduced lung function based on $\mathrm{FEV}_{1}$ or $D_{\mathrm{LCO}}<80 \%$ of predicted. $D_{\mathrm{LCO}}$ was lower in hospitalised compared to outpatients $(70 \% \pm 18$ predicted and $80 \% \pm 11$ predicted, respectively, $\mathrm{p}=0.023)$. This holds true when comparing admitted patients with and without oxygen therapy requirement during acute COVID-19 (66\% and 79\%, p=0.004). The group means of $\mathrm{FEV}_{1}$ were normal $(>80 \%$ predicted) for all patients also when divided into hospitalised and not hospitalised, but lower in the patients who had been hospitalised $(\mathrm{p}=0.024)$

Exercise capacity was also evaluated by having the patients perform the 1-MSTST and maximal handgrip. As seen in table 3, almost half of patients (44\%) performed below the 25th percentile (compared to 


\begin{tabular}{|c|c|c|c|c|}
\hline & Overall & Non-hospitalised & Hospitalised & $\begin{array}{l}\text { p-value } \\
\text { hospitalised versus } \\
\text { non-hospitalised }\end{array}$ \\
\hline Demographics & 57 & 23 & 34 & \\
\hline Age years & $51(13)$ & $41(12)$ & $57(10)$ & $<0.001$ \\
\hline Male sex $n(\%)$ & $28(49)$ & $7(30)$ & $21(61)$ & 0.040 \\
\hline Race or ethnic group & & & & 0.206 \\
\hline Caucasian n (\%) & $44(77)$ & $19(83)$ & $25(74)$ & \\
\hline Asian n (\%) & $2(4)$ & $2(9)$ & $0(0.0)$ & \\
\hline African n (\%) & $2(4)$ & $0(0.0)$ & $2(6)$ & \\
\hline Indian n (\%) & $1(2)$ & $0(0.0)$ & $1(3)$ & \\
\hline Middle Eastern n (\%) & $8(14.0)$ & $2(8.7)$ & $6(18)$ & \\
\hline $\mathrm{BMI} \mathrm{kg} \cdot \mathrm{m}^{-2}$ & 27 & 26 & 29 & 0.095 \\
\hline $\mathrm{BMI}>30 \mathrm{~kg} \cdot \mathrm{m}^{-2} \mathrm{n}(\%)$ & $12(26)$ & $3(14)$ & $9(36)$ & 0.182 \\
\hline Smoking & & & & 0.442 \\
\hline Current smoker n (\%) & $1(2)$ & $1(4)$ & $0(0.0)$ & \\
\hline Previous smoker n (\%) & $22(39)$ & $8(35)$ & $14(41)$ & \\
\hline Time since cessation years & $17(15)$ & $18(11)$ & $17(17)$ & 0.901 \\
\hline Work status & & & & 0.023 \\
\hline Working n (\%) & $45(79)$ & $22(96)$ & $23(68)$ & \\
\hline Currently out of work n (\%) & $3(5)$ & $1(4)$ & $2(6)$ & \\
\hline Retired n (\%) & $9(16)$ & $0(0.0)$ & $9(27)$ & \\
\hline \multicolumn{5}{|l|}{ Comorbidities } \\
\hline Charlson Morbidity Index & $2.26(2.26)$ & $1.00(1.00)$ & $3.12(2.47)$ & $<0.001$ \\
\hline Diabetes mellitus n (\%) & $5(9)$ & $0(0.0)$ & $5(15)$ & 0.148 \\
\hline Moderate to severe CKD n (\%) & $2(4)$ & $0(0.0)$ & $2(6)$ & 0.652 \\
\hline COPD n (\%) & $2(4)$ & $0(0.0)$ & $2(6)$ & 0.652 \\
\hline Asthma n (\%) & $15(26)$ & $4(17)$ & $11(32)$ & 0.341 \\
\hline \multicolumn{5}{|l|}{ Acute COVID-19 severity } \\
\hline Mean days in the hospital days & - & 0 & $13.3(21.9)$ & - \\
\hline Oxygen therapy $n(\%)$ & - & 0 & $21(61.8)$ & - \\
\hline $\begin{array}{l}\text { Maximum oxygen } L \cdot \min ^{-1} \text { of those } \\
\text { on oxygen }\end{array}$ & - & - & $20(19.6)$ & - \\
\hline Oxygen $>0$ and $<5 \mathrm{~L} \cdot \mathrm{min}^{-1} \mathrm{n}(\%)$ & - & - & $7(20.6)$ & - \\
\hline Oxygen $>5$ and $<15 \mathrm{~L} \cdot \mathrm{min}^{-1} \mathrm{n}(\%)$ & - & - & $4(11.8)$ & - \\
\hline HFNC n (\%) & - & - & $9(26.5)$ & - \\
\hline Intubation n (\%) & - & - & $1(2.9)$ & - \\
\hline Maximum ferritin $\mu \mathrm{g} \cdot \mathrm{L}^{-1}$ & - & NA & $1340(440-2295)$ & - \\
\hline Minimum lymphocytes $\times 10^{9} \cdot \mathrm{L}^{-1}$ & - & NA & $0.9(0.7-1.3)$ & - \\
\hline Maximum CRP $\mathrm{mg} \cdot \mathrm{L}^{-1}$ & - & NA & $153(84-203)$ & - \\
\hline Maximum LDH U. $\mathrm{L}^{-1}$ & - & NA & $376(259-531)$ & - \\
\hline Procalcitonin $\mu \mathrm{g} \cdot \mathrm{L}^{-1}$ & - & NA & $0.16(0.10-0.66)$ & - \\
\hline Maximum D-dimer FEU $\mathrm{L}^{-1}$ & - & NA & $0.87(0.72-1.65)$ & - \\
\hline $\begin{array}{l}\text { Data is presented as mean (standard } \\
\text { BMI: body mass index; CKD: chronic k } \\
\text { CRP: C-reactive protein; LDH: lactate d }\end{array}$ & $\begin{array}{l}\text { iation) or } \\
\text { ey diseas } \\
\text { ydrogenas }\end{array}$ & $\begin{array}{l}\text { (percentage). } \\
\text { HFNC: high-flow } \\
\text {; NA: not available }\end{array}$ & al continuous $p$ & tive airway pressure; \\
\hline
\end{tabular}

population-based reference values [28]) in the 1-MSTST, and almost one-third of patients (28\%) performed below the 25th percentile in handgrip dynamometry (compared to population-based reference values [29]).

CPET was performed in a subset of 31 patients. Overall maximal oxygen uptake $\left(V^{\prime} \mathrm{O}_{2} \mathrm{max}\right)$ was 27.5 (8.9) $\mathrm{kg} \cdot \mathrm{mL} \cdot \mathrm{min}^{-1}$, and only two patients had a $V^{\prime} \mathrm{O}_{2} \max <80 \%$ predicted, while 11 patients had a breathing reserve of $<25 \%$. CPET test was considered normal in $50 \%$ of all patients. Although not significant, it was more often abnormal in the participants that had been hospitalised and requiring oxygen. In all patients it was either decreased physical fitness $(\mathrm{N}=11,35.5 \%)$ or decreased ventilatory capacity $(\mathrm{N}=5,16 \%)$ that was the main reason for their abnormal CPET.

High-resolution computed tomography of the chest

In figure 3 we present HRCT data at 3 months follow-up. Overall, 53\% of patients had abnormalities on HRCT, and the median total HRCT score was 0.5 (0.0-3.8); hospitalised patients had a higher CT score 


\begin{tabular}{|c|c|c|c|c|}
\hline & Overall & Non-hospitalised & Hospitalised & $\mathrm{p}$-value \\
\hline Reported complete restitution & $5(8.8)$ & $1(4.3)$ & $4(11.8)$ & 0.621 \\
\hline MRC dyspnoea score & $2(2-3)$ & $2(2-3)$ & $2(2-2)$ & 0.106 \\
\hline CAT score & $12(8-18)$ & $16(10-24)$ & $11(8-16)$ & 0.069 \\
\hline Cough & $1(1-2)$ & $1(1-2)$ & $1(1-2)$ & 0.662 \\
\hline Phlegm & $1(0-2)$ & $1(0-2)$ & $1(0-2)$ & 0.552 \\
\hline Chest pain & $2(0-3)$ & $3(2-4)$ & $1(0-2.5)$ & 0.001 \\
\hline Breathlessness walking a hill & $3(2-4)$ & $3.5(3-5)$ & $2(1-3)$ & 0.001 \\
\hline Limited in activities at home & $1(0-3)$ & $2.5(1-3)$ & $1(0-2)$ & 0.125 \\
\hline Confident in leaving home & $0(0-1)$ & $0(0-1)$ & $0(0-1)$ & 0.735 \\
\hline I sleep soundly & $2(1-3)$ & $2.5(0.25-3)$ & $2(1-2)$ & 0.365 \\
\hline I have lots of energy & $3(2-4)$ & $4(2.25-4)$ & $3(1.5-4)$ & 0.034 \\
\hline \multicolumn{5}{|l|}{ EQ-5D-5L } \\
\hline Index value & $0.74(0.65-0.86)$ & $0.74(0.66-0.80)$ & $0.79(0.65-0.86)$ & 0.646 \\
\hline EQ-5D VAS (0-100) & $70(55-81)$ & $65(55-79)$ & 75 (59-90) & 0.206 \\
\hline PCFS grade after COVID & $2(1-2)$ & $2(2-2)$ & $1(1-3)$ & 0.145 \\
\hline \multicolumn{5}{|l|}{ WPAI:GH } \\
\hline$\%$ work time missed due to health & $0(0-24)$ & $10(0-41)$ & $0(0-0)$ & 0.053 \\
\hline$\%$ impairment while working due to health & $20(9-45)$ & $35(20-60)$ & $10(1-28)$ & 0.095 \\
\hline$\%$ overall work impairment due to health & $23(6-66)$ & $54(20-70)$ & $10(0.00-27.95)$ & 0.024 \\
\hline$\%$ activity impairment due to health & $30(10-60)$ & $45(30-78)$ & $10(8-55)$ & 0.020 \\
\hline
\end{tabular}

than non-hospitalised (2.3 (0.1-4.8) and $0.0(0.0-0.3), \mathrm{p}<0.001)$. The most common pathology was ground-glass opacities, which was significantly higher in hospitalised than non-hospitalised patients $(\mathrm{p}<0.001)$.

\section{Cardiac-autonomic evaluation}

Cardiac evaluation (history, pro-brain natriuretic peptide (pro-BNP), 12-lead ECG high-resolution ECG) was normal in all patients but one, who presented with chest discomfort at 3 months follow-up. He was diagnosed with non-ST-elevation myocardial infarction and underwent revascularisation with stent implantation.

Participants had a normal median pro-BNP 5.3 (2.7-11.6) with no intergroup differences. Sinus rhythm was present in all patients but one in the hospitalised group who was known with atrial fibrillation prior to

TABLE 3 Lung function and functional tests at 3-months follow-up

\begin{tabular}{|c|c|c|c|c|}
\hline & Overall & Non-hospitalised & Hospitalised & $\mathrm{p}$-value \\
\hline \multicolumn{5}{|l|}{ Lung function testing } \\
\hline $\mathrm{FEV}_{1} \%$ pred & $90(16)$ & $96(13)$ & $86(17)$ & 0.024 \\
\hline FVC $\%$ pred & $89(17)$ & $97(14)$ & $84(17)$ & 0.002 \\
\hline $\mathrm{FEV}_{1} / \mathrm{FVC} \%$ & $81(7)$ & $82(7)$ & $81(8)$ & 0.835 \\
\hline$D_{\text {LCO }} \%$ pred & $74(16)$ & $80(11)$ & $70(18)$ & 0.023 \\
\hline DCLO/VA \% pred & $89(16)$ & $87(11)$ & $90(18)$ & 0.637 \\
\hline 1-MSTS & & & & 0.474 \\
\hline$>25$ th percentile $\mathrm{n}(\%)$ & $30(52.6)$ & $12(52.2)$ & $18(52.9)$ & 0.792 \\
\hline$\leqslant 25$ th percentile $\mathrm{n}(\%)$ & $25(43.9)$ & $11(47.8)$ & $14(41.2)$ & - \\
\hline Max handgrip & & & & 0.294 \\
\hline >25th percentile $\mathrm{n}(\%)$ & $39(68.4)$ & $18(78.3)$ & $21(61.8)$ & 0.737 \\
\hline$\leqslant 25$ th percentile $\mathrm{n}(\%)$ & $16(28.1)$ & $5(21,7)$ & $11(32.4)$ & - \\
\hline
\end{tabular}

$\mathrm{FEV}_{1}$ : forced expiratory volume in $1 \mathrm{~s}$; FVC: forced vital capacity; $D_{\mathrm{Lco}}$ : diffusing capacity of the lung for carbon monoxide; DCLO/VA: diffusion capacity/alveolar volume; 1-MSTS: 1-min sit and stand. 
Hospitalised Non-hospitalised

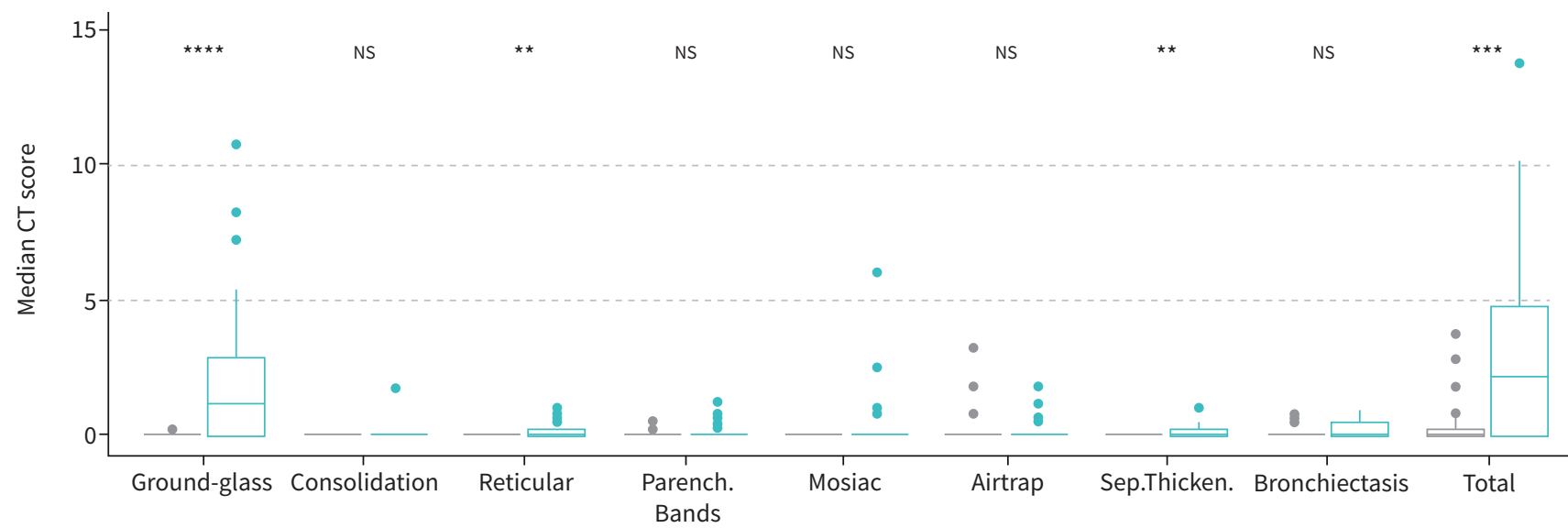

FIGURE 3 High-resolution computed tomography (HRCT) findings in non-hospitalised and hospitalised. Median computed tomography (CT) scores for the different radiological entities with comparison between non-hospitalised and hospitalised patients (a) or non-hospitalised, hospitalised without necessity for oxygen treatment and hospitalised with oxygen treatment (b), respectively. Ground-glass: ground-glass opacities; Consolidation: airspace consolidation; Reticular: reticular pattern; Parench.Bands: pleuraparenchymal bands; Airtrap: air trapping; Sep.Thicken: interlobular septal thickening; Bronchiec: traction bronchiectasis and traction bronchiolectasies. ${ }^{\star \star}: \mathrm{p}<0.01 ;{ }^{\star \star \star}: \mathrm{p}<0.001 ;{ }^{\star \star \star \star}: \mathrm{p}<0.0001$.

COVID-19. Evaluation of autonomic function showed DC and periodic repolarisation dynamics (PRD) in normal range for both groups.

\section{Cognitive functions}

Objective performance-based and subjective self-reported cognition data are presented in table 4 .

The percentage of patients with clinically significant cognitive impairment ranged from 51\% to 58\%, depending on the cut-off scores implemented for determining "clinically relevant" impairment. Of these, 38-53\% of patients showed broad impairments (performance decline on a global cognition measure), and an additional $4-16 \%$ patients showed selective impairments (clinically relevant impairment on $\geqslant 2$ of the 6 tests). The frequency of cognitive impairments ranged from $59 \%$ to $66 \%$ for hospitalised patients and $31 \%$ to $44 \%$ for non-hospitalised patients depending on the chosen cut-off. Further detail on cognitive results is provided in supplementary results.

\section{Correlation COVID-19 severity with follow-up abnormalities}

Correlations are depicted in figure 4. At 3-month follow-up, symptom scores (CAT, MRC) were not correlated with lung function (spirometry and diffusion capacity) or HRCT data. However, post-COVID-19, HRCT total score was associated with $\mathrm{FEV}_{1}(R=-0.49 ; \mathrm{p}<0.001)$ and $D_{\mathrm{LCO}}$ impairments $(R=-0.61 ; \mathrm{p}<0.001)$. Moreover, post-COVID-19, $\mathrm{FEV}_{1}, D_{\mathrm{LCO}}$ and HRCT abnormalities were significantly associated with hospitalisation duration and maximum oxygen requirement during acute COVID-19 illness. EQ5D-5 L scores were significantly correlated to self-reported symptoms of dyspnoea by CAT and MRC, post-covid performance status by PCFS and self-reported cognitive status.

\section{Discussion}

We performed a multidisciplinary evaluation of 57 patients 3 months after their COVID-19 illness for possible physical, cognitive and social complications. Only a small percentage of patients reported complete subjective recovery. All 34 initially hospitalised patients and 22 out of 23 non-hospitalised patients had at least one complaint or abnormal finding 3 months post COVID-19. Most noteworthy, in the cohort of hospitalised patients, was that $D_{\text {LCO }}$ decreased below normal and HRCT abnormalities were found in about half of patients with the most common abnormality being ground-glass opacities.

Impaired lung function has been shown to persist in survivors of COVID-19 pneumonia with impaired $D_{\text {LCO }}$ being the most common abnormality [7, 30], reflecting the pathophysiological changes seen in acutely ill patients. $\mathrm{FEV}_{1}$ in our patients was on average normal but significantly different between hospitalised versus non-hospitalised patients and is in accordance with a study of 57 patients evaluated 30 
TABLE 4 Objective and subjective measures of cognition from the subsample of 45 patients with available cognition data

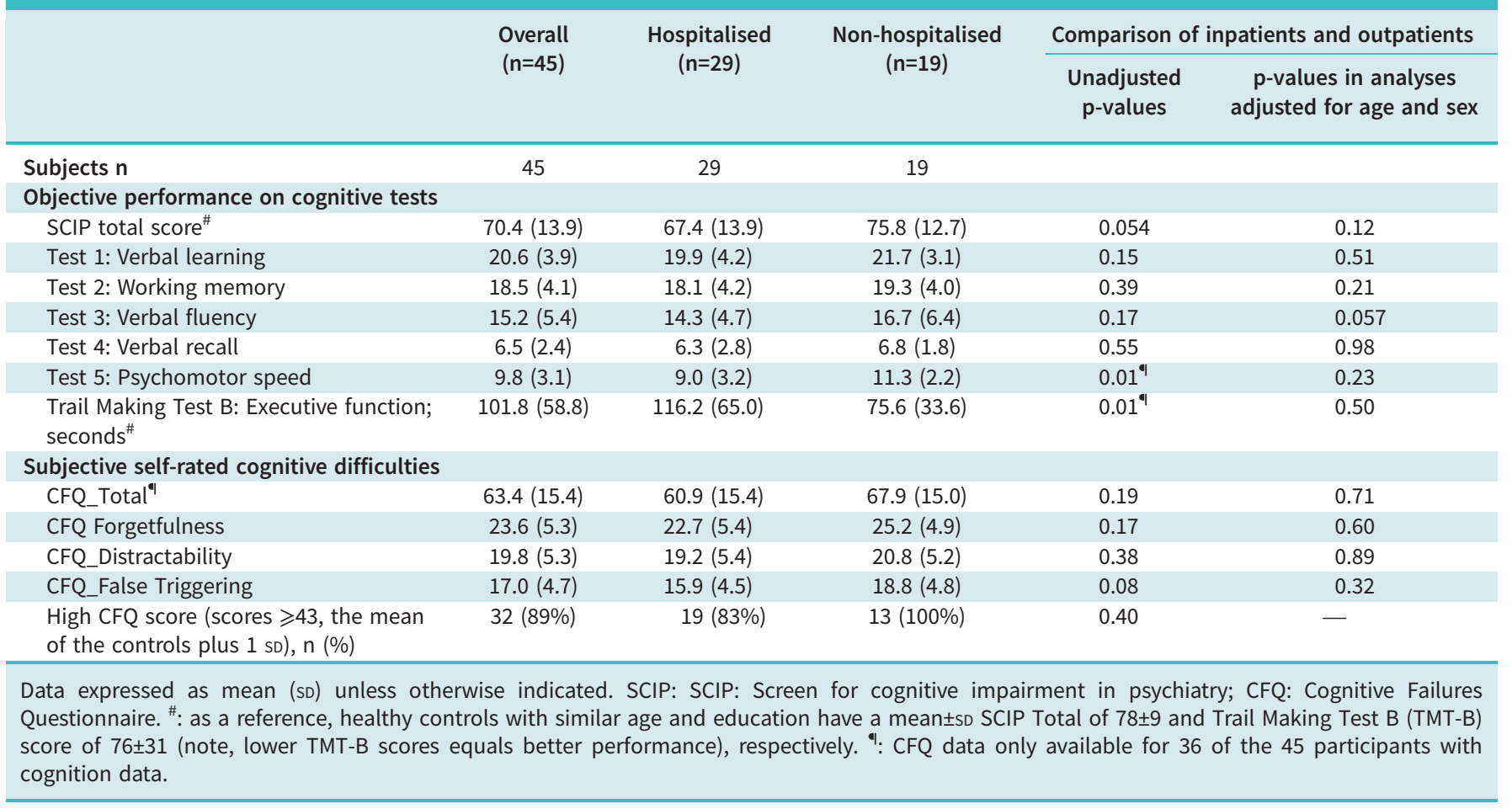

days after their COVID-19 disease [31]. Our data suggest lung function impairment even 3 months after COVID-19 and an association with hospitalisation, duration of hospitalisation and oxygen requirement.

We found that hospitalisation and oxygen support resulted in radiological abnormalities at 3 months follow-up. Hospitalised patients had higher CT-scores, reflecting more abnormalities, than non-hospitalised patients that were still present 3 months after COVID-19. Only one patient in the hospitalised group who had been intubated presented with fibrosis at follow-up. Our finding of predominately ground-glass opacities is in line with a recent study including 103 patients that reported ground-glass opacities in one out of four patients 3 months after COVID-19 [9]. Five patients in the hospitalised group showed mosaic attenuation, which may be indicative of perfusion defects. However, only slightly increased d-dimer values were found at the index stay, and two patients underwent a CT scan with contrast that excluded thrombosis. It has been hypothesised that pulmonary pathology in COVID-19 is in fact primarily a vascular thrombotic disease with microemboli being the driver of COVID-19-related acute respiratory distress syndrome [32]. In one study pulmonary Dual Energy CT (DECT) angiography revealed a significant number of pulmonary ischaemic areas even in the absence of visible pulmonary arterial thrombosis [3] which may reflect microthrombosis associated with COVID-19 pneumonia.

In our study we tested physical capacity by functional tests in the form of 1-MSTST, maximum handgrip and CPET (the latter in a subset of 31 patients) as decrease in these measures has been shown to be associated with mortality and quality of life in certain patient groups [33] and has been used to assess exercise-induced desaturation in patients with different pulmonary pathologies including interstitial pulmonary disease [34]. In short about half of patients doing 1-MSTS and one-third of patients doing the handgrip dynamometric test performed below the 25th percentile of an age- and sex-matched population, and this was independent of whether or not patients had been hospitalised. In line with this, only a small fraction of the patients had severe reduced ventilator capacity as measured by CPET, while a larger fraction had reduced breathing reserve or deconditioning. We know from the previous SARS epidemic that patients had impairment in these tests primarily because of physical deconditioning [35]. Collectively, most studies find decreased physical function after COVID-19 illness at some level. Correspondingly, a low level of physical capacity was detected in an Italian cohort of COVID-19 patients using 1-MSTST among other tests [36]. In another recent study evaluating 124 patients at 3 months after COVID-19, although they did not find that patients had significantly decreased physical capacity evaluated with the 6-min walk distance (6MWD), they did find that 22\% of patients had low 6MWD [37]. 


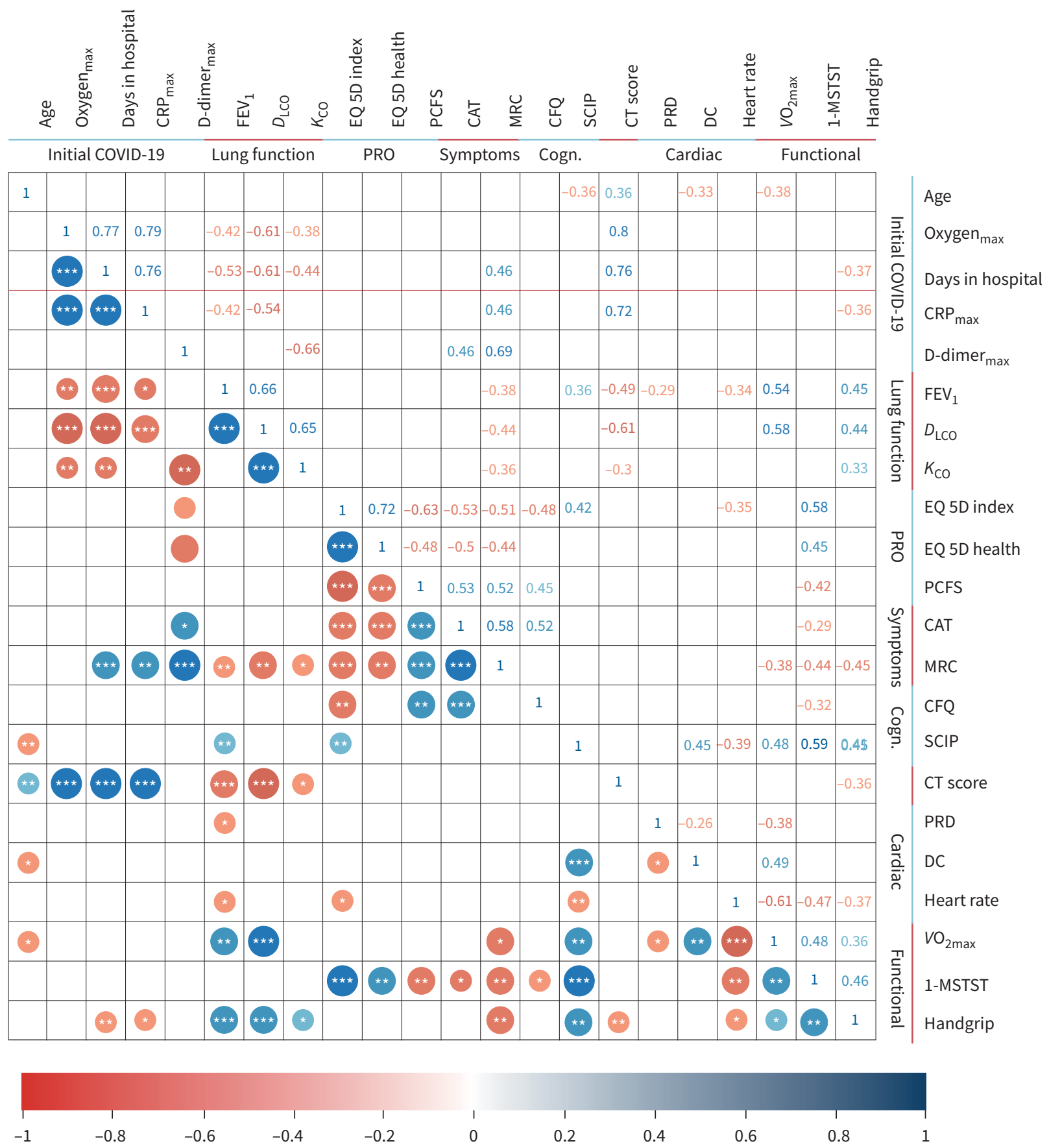

FIGURE 4 Correlations between intitial covid, lung function, patient-reported outcomes, symptoms, cognitive measures, functional tests, cardiac tests and cognitive measures. Pearson correlations for coronavirus disease 2019 (COVID-19), lung function, patient-reported outcomes (PRO), symptoms, cognition (Cogn.), cardiac and functional. Only correlation coefficients with $p<0.05$ are shown. ${ }^{*} p<0.05 ;{ }^{\star \star} p<0.01 ;{ }^{\star \star \star} p<0.001$. CRP: C-reactive protein; $\mathrm{FEV}_{1}$ : forced expiratory volume in $1 \mathrm{~s}$; $D_{\mathrm{LCO}}$ : diffusing capacity of the lung for carbon monoxide; $K_{\mathrm{CO}}$ : carbon monoxide transfer coefficient; PCFS: post-COVID functional scale; CAT: COPD Assessment Test; MRC: Medical Research Council dyspnoea score; CFQ: Cognitive Failures Questionnaire; SCIP: screen for cognitive impairment in psychiatry; EQ-5D: 5 Dimension 5 Level Quality of Life Questionnaire; EQ-5D Health/ VAS: 5 Dimension 5 Level Quality of Life Questionnaire visual analog scale; CT: computed tomography; PRD: periodic repolarisation dynamics; DC: deceleration capacity; $V^{\prime} O_{2}$ max: maximal oxygen uptake; 1-MSTST: 1-min sit and stand test.

Patients in this study displayed objective performance difficulties on the cognitive tests, with $51-58 \%$ being clinically significant. Notably, subjective cognitive difficulties did not differ between hospitalised and non-hospitalised patients. Although the sample size of our patient cohort was moderate, this suggests 
that cognitive consequences of COVID-19 illness may not only be limited to the most severely affected patients. In keeping with this, internet-based testing of cognitive functions in $>80000$ people with suspected or biologically confirmed COVID-19 revealed most pronounced cognitive impairments in people who had been hospitalised with COVID-19 illness, but also mild impairments in biologically confirmed cases with no reported breathing difficulties [38]. Additionally, $>90 \%$ of our patient cohort presented with moderate to severe self-reported cognitive difficulties. This is in line with previous evidence for long-term subjective cognitive sequelae of COVID-19 [8]. Our findings thus add to the emerging evidence for cognitive consequences of COVID-19 in both hospitalised and non-hospitalised patients that may persist for several months and impede return to work and daily functioning.

The most common persistent symptoms that patients in our study reported was dyspnoea and chest discomfort. Accordingly, an Italian study reported that $87 \%$ of people discharged from hospital still experienced at least one symptom 2 months after their initial illness; the more common symptoms being fatigue, difficulty breathing, and joint and chest pain [6]. Correspondingly GoËRTZ et al. [39] also found only partial resolution of symptoms 3 months after COVID-19 infection in a sample of previously hospitalised and non-hospitalised patients.

\section{Limitations}

Our study has some limitations. First, this was an observational, cross-sectional study at 3 months after COVID-19. Patients' conditions before COVID-19 were often unknown, and clinical data during the initial hospital stay was only compiled to an extent as clinically necessary and systematically available from hospital records. It is possible that the abnormalities we find are unrelated to patients' COVID-19 illness and may have been pre-existent. Furthermore, progression or regression of long-term consequences cannot be evaluated yet. Secondly, the study suffers from a selection bias, as non-hospitalised patients were referred by their general physician because of symptoms. On the other hand, sicker and frail patients may have refrained from study participation due to severe symptoms or fatigue. In addition, clinical and laboratory data from non-hospitalised patients during the acute phase were not available. Thirdly, we are aware that our patient cohort is small in size. However, patients in this study are very well characterised in terms of their post-covid clinical status and complications, using a broad variety of objective tests. We considered these data important to report in order to raise awareness of the very large number of patients that may suffer from long COVID (also in primary care) and to offer the most suitable screening and follow-up programme for long COVID patients.

\section{Conclusion}

In conclusion, we demonstrate that 3 months after COVID-19, the majority of patients showed signs of organ function impairment, underlining the importance of multidimensional management strategies in these patients. Most noteworthy, impairment was seen in cognition and quality of life regardless of hospitalisation and alterations in HRCT of the chest and impaired $D_{\text {LCO }}$ that were present in hospitalised patients. Patients, even though not hospitalised, may still experience reduction in quality of life and perceived health. An important outcome of the data presented here is that they have contributed to the establishment of post-COVID-19 clinics on a national basis as well as formed our standard of care follow-up programme.

Conflict of interest: S. Johnsen has nothing to disclose. S.M. Sattler has nothing to disclose. K.W. Miskowiak reports personal feels from Lundbeck outside the submitted work. K. Kunalan has nothing to disclose. A. Victor has nothing to disclose. L. Pedersen has nothing to disclose. H.F. Andreassen has nothing to disclose. B.J. Jørgensen has nothing to disclose. H. Heebøll has nothing to disclose. M.B. Andersen has nothing to disclose. L. Marner has nothing to disclose. C. Hædersdal has nothing to disclose. H. Hansen has nothing to disclose. S. Ditlev has nothing to disclose. C. Porsbjerg has nothing to disclose. T.S. Lapperre has nothing to disclose.

\section{References}

1 Holler JG Eriksson R, Jensen TO, et al. First wave of COVID-19 hospital admission in Denmark: a Nationwide population-based cohort study. BMC Infect Dis 2021; 21: 39.

2 Zhu H, Rhee JW, Cheng P, et al. Cardiovascular complications in patients with COVID-19: consequences of viral toxicities and host immune response. Curr Cardiol Rep 2020; 22: 32.

3 Grillet F, Busse-Coté A, Calame P, et al. COVID-19 pneumonia: microvascular disease revealed on pulmonary dual-energy computed tomography angiography. Quant Imaging Med Surg 2020; 10: 1852-1862.

4 Huang X, Wei F, Hu L, et al. Epidemiology and clinical characteristics of COVID-19. Arch Iran Med 2020; 23: 268-271.

5 Hui DS, Wong KT, Ko FW, et al. The 1-year impact of severe acute respiratory syndrome on pulmonary function, exercise capacity, and quality of life in a cohort of survivors. Chest 2005; 128:2247-2261. 
6 Carfi A, Bernabei R, Landi F. Persistent symptoms in patients after acute COVID-19. J Am Med Assoc 2020; 324: 603-605.

7 George PM, Barratt SL, Condliffe R, et al. Respiratory follow-up of patients with COVID-19 pneumonia. Thorax 2020; 75:1009-1016.

8 Lambert NJ, Survivor Corps. COVID-19 "Long Hauler" Symptoms Survey Report. Online Survey. Indiana University School of Medicine, 2020. Available from: https://dig.abclocal.go.com/wls/documents/2020/ 072720-wls-covid-symptom-study-doc.pdf

9 Lerum TV, Aaløkken TM, Brønstad E, et al. Dyspnoea, lung function and CT findings three months after hospital admission for COVID-19. Eur Respir J 2020; 57: 2003448.

10 Greenhalgh T, Knight M, A'Court C, et al. Management of post-acute covid-19 in primary care. BMJ 2020; 370: m3026.

11 Guler SA, Ebner L, Beigelman C, et al. Pulmonary function and radiological features four months after COVID-19: first results from the national prospective observational Swiss COVID-19 lung study. Eur Respir $J$ 2021; 7: 2003690.

12 Anastasio F, Barbuto S, Scarnecchia E, et al. Medium-term impact of COVID-19 on pulmonary function, functional capacity and quality of life. Eur Respir J 2021.

13 Qin W, Chen S, Zhang Y, et al. Diffusion capacity abnormalities for carbon monoxide in patients with COVID-19 at three-month follow-up. Eur Respir J 2021; 58: 2003677.

14 López-León S, Wegman-Ostrosky T, Perelman C, et al. More than 50 long-term effects of COVID-19: a systematic review and meta-analysis. medRxiv 2021; preprint [doi: 10.1101/2021.01.27.21250617].

15 Huang C, Huang L, Wang Y, et al. 6-month consequences of COVID-19 in patients discharged from hospital: a cohort study. Lancet 2021; 397: 220-232.

16 Avula A, Nalleballe K, Narula N, et al. COVID-19 presenting as stroke. Brain Behav Immun 2020; 87: 115-119.

17 Oxley TJ, Mocco J, Majidi S, et al. Large-vessel stroke as a presenting feature of Covid-19 in the young. $N$ Engl J Med 2020; 382: e60.

18 Lennon JC. Neurologic and immunologic complications of COVID-19: potential long-term risk factors for Alzheimer's disease. J Alzheimer's Dis Rep 2020; 4: 217-221.

19 EuroQol Research Foundation. EQ-5D-5 L - EQ-5D. EQ-5D; 2019. https://euroqol.org/ Date last accessed: 18 Jan 2021.

20 Klok FA, Boon GJAM, Barco S, et al. The post-COVID-19 functional status scale: a tool to measure functional status over time after COVID-19. Eur Respir J 2020; 56: 2001494.

21 Broadbent DE, Cooper PF, FitzGerald P, et al. The cognitive failures questionnaire (CFQ) and its correlates. Br J Clin Psychol 1982; 21: 1-16.

22 Brusselle GG, Gaga M. ERS guidelines, statements and technical standards published in the ERJ in 2014: a year in review. Eur Respir J 2015; 45: 863-866.

23 Zanini $\mathrm{A}$, Aiello $\mathrm{M}$, Cherubino $\mathrm{F}$, et al. The one repetition maximum test and the sit-to-stand test in the assessment of a specific pulmonary rehabilitation program on peripheral muscle strength in COPD patients. Int J Chron Obstruct Pulmon Dis 2015; 10: 2423-2430.

24 Albouaini K, Egred M, Alahmar A, et al. Cardiopulmonary exercise testing and its application. Postgrad Med J 2007; 83: 675-682.

25 Søyseth V, Aaløkken TM, Mynarek G, et al. Diagnosis of biopsy verified usual interstitial pneumonia by computed tomography. Respir Med 2015; 109: 897-903.

26 Jensen JH, Støttrup MM, Nayberg E, et al. Optimising screening for cognitive dysfunction in bipolar disorder: validation and evaluation of objective and subjective tools. J Affect Disord 2015; 187: 10-19.

27 War Department, Adjutant General's Office. Army Individual Test Battery. Manual of Directions and Scoring. Washington, DC, War Department, Adjutant General's Office, 1994.

28 Strassmann A, Steurer-Stey C, Lana KD, et al. Population-based reference values for the 1-min sit-to-stand test. Int J Public Health 2013; 58: 949-953.

29 Schlüssel MM, dos Anjos LA, de Vasconcellos MTL, et al. Reference values of handgrip dynamometry of healthy adults: a population-based study. Clin Nutr 2008; 27: 601-607.

30 Fraser E. Long term respiratory complications of covid-19. BMJ (Clinical research ed.) 2020; 370: m3001.

31 Huang Y, Tan C, Wu J, et al. Impact of coronavirus disease 2019 on pulmonary function in early convalescence phase. Respir Res 2020; 21: 163.

32 Ackermann M, Verleden SE, Kuehnel M, et al. Pulmonary vascular endothelialitis, thrombosis, and angiogenesis in Covid-19. N Engl J Med 2020; 383: 120-128.

33 Crook S, Frei A, ter Riet G, et al. Prediction of long-term clinical outcomes using simple functional exercise performance tests in patients with COPD: a 5-year prospective cohort study. Respir Res 2017; 18: 112.

34 Briand J, Behal $\mathrm{H}$, Chenivesse $\mathrm{C}$, et al. The 1-minute sit-to-stand test to detect exercise-induced oxygen desaturation in patients with interstitial lung disease. Ther Adv Respir Dis 2018; 12: 1753466618793028.

35 Ngai JC, Ko FW, Ng SS, et al. The long-term impact of severe acute respiratory syndrome on pulmonary function, exercise capacity and health status. Respirology 2010; 15: 543-550. 
Belli S, Balbi B, Prince I, et al. Low physical functioning and impaired performance of activities of daily life in COVID-19 patients who survived hospitalisation. Eur Respir J 2020; 56: 2002096.

37 van den Borst $\mathrm{B}$, Peters JB, Brink $\mathrm{M}$, et al. Comprehensive health assessment three months after recovery from acute COVID-19. Clin Infect Dis 2020; in press [doi:10.1093/cid/ciaa1750].

38 Hampshire A, Trender W, Chamberlain SR, et al. Cognitive deficits in people who have recovered from COVID-19 relative to controls: an $N=84285$ online study. medRxiv 2020; preprint [doi:10.1101/2020.10.20. 20215863].

39 Goërtz YMJ, van Herck M, Delbressine JM, et al. Persistent symptoms 3 months after a SARS-CoV-2 infection: the post-COVID-19 syndrome? ERJ Open Res 2020; 6: 00542-2020. 